\title{
EFECTOS DE LAS REPRESENTACIONES SOCIALES DE LAS MAMAS SOBRE LA PREVENCIÓN DEL CÁNCER MAMARIO EN UN GRUPO DE MUJERES COLOMBIANAS
}

\author{
Clara Victoria Giraldo Mora \\ MARIA EUGENIA ARANGO ROJA \\ Universidad de Antioquia
}

Recibido/15/07/2011

Aceptado/25/09/2011

\section{Resumen}

Este escrito presenta los resultados de un estudio para comprender las representaciones sociales de las mamas y su influencia en el diagnostico precoz del cáncer mamario y las prácticas de auto cuidado en un grupo de mujeres de Medellín, Colombia. Es un estudio cualitativo llevado a cabo con 19 entrevistas en profundidad a 19 mujeres adultas sin cáncer de mama, utilizando el criterio de máxima variación. El análisis utiliza los lineamientos de la teoría fundamentada. Los hallazgos muestran que existe una representación social de las mamas como objeto de atracción y una representación negativa del cáncer de mama, que no favorecen la prevención del cáncer de mama ni contribuyen a las prácticas de auto cuidado como el auto examen, el tamizaje clínico y la mamografía. Concluimos que existe una construcción problemática del cuerpo femenino entre las participantes que impide las prácticas que merece ser revisada y denunciada como un asunto político esencial en la construcción de la subjetividad femenina.

Palabras clave: cuerpo, imagen corporal, belleza, autocuidado, cancer de mama, género, salud, representaciones sociales y estética.

\footnotetext{
Abstract

This paper presents the results of a study to understand the social representations of breast cancer and how they influence breast cancer prevention and self-care practices
} 
in a group of women from Medellin-Colombia. Is a qualitative study carried out with 19 in-depth interviews to 19 adult women who have not had breast cancer and using the sampling criterion of maximum variation. In our analysis, we used the guidelines of Grounded Theory. The findings show that there is a social representation of breasts as object of attraction and a negative representation of breast cancer, none of these representations foster breast cancer prevention nor contribute to self-care practices such as breast self-examination, clinical breast screening and mammography. In synthesis, there are problematic constructions of the feminine body among participants that deserve to be revised and denounced as political issue essential to the construction of feminine subjectivity .

Keywords: Body, Body Image, Beauty, Self-care, Brest Cancer, Gender and Health. 


\section{Introducción}

Anualmente, se presentan en el mundo más de 10 millones de casos nuevos de cáncer de mama y más de 6 millones mueren a causa de esta enfermedad, que suponen el $12 \%$ del total de muertes en la población femenina. Hace veinte años, estas cifras eran de 6 y 4 millones respectivamente ${ }^{1}$ y se estima que los casos aumentarán a 15 millones en el 2020². Estas cifras muestran el cáncer de mama como el más frecuente entre la población femenina y un problema de salud pública que afecta a una de cada diez mujeres.

En Colombia, el cáncer es un problema relevante en el perfil epidemiológico $^{3}$. Según el Instituto Nacional de Cancerología, en el año 2002 el cáncer ginecológico representó el 19,6\% de los casos, siendo el de mama el segundo más común en mujeres (19,7\%). El 70\% de los casos fue diagnosticado en estadios tardíos (III y IV) y el $5,1 \%$ en estadios tempranos (in situ y I) ${ }^{4}$. A pesar de ello, el control de este cáncer no es una prioridad en la Política Nacional de Salud Sexual y Reproductiva, diseñada por el Ministerio de la Protección Social en el $2003^{5}$.

El auto examen de mama y la mamografía constituyen las estrategias más importantes de prevención y detección temprana de la enfermedad y la mejor alternativa para controlarlo, así como para prevenir y detectar las recurrencias y las metástasis a otros órganos. Es preocupante saber que estas acciones no

1. ORGANIZACIÓN MUNDIAL DE LA SALUD. «Programas Nacionales de Control del Cáncer: Políticas y Pautas para la Gestión». Organización Mundial de la Salud. <http:// www.paho.org/Spanish/AD/DPC/NC/pcc-NCCP.pdf>. Consultado el 7-08-2005.

2. RADICE, D., \& REDAELLI, A. Breast cancer management: quality of life and cost considerations. Harmacoeconomics 21 (2003), pp. 383-396.

3. Pardo, C., Murillo. R., Piñeros. M., y Castro, M. Casos nuevos de cáncer 2003 en el Instituto Nacional de Cancerología Colombia, Revista Colombiana de Cancerología, 7 (2003). p. 5.

4. MINISTERIO DE SALUD DE COLOMBIA. «III Estudio Nacional de Salud Bucal y II Estudio Nacional de factores de riesgo de enfermedades crónicas- ENFERC II.» Colombia. El Ministerio; Serie Documentos Técnicos. (1999) p. 31.

5. MINISTERIO DE LA PROTECCIÓN SOCIAL COLOMBIA. «Política Nacional de Salud Sexual y Reproductiva.» Bogotá. El Ministerio, (2003). Documentos técnicos p. 11.

Feminismo/s 18, diciembre 2011, pp. 249-270 
se realizan en nuestro medio con la frecuencia recomendada ${ }^{6}$, lo que se evidencia en el estudio nacional de factores de riesgo de enfermedades crónicas realizado por el Ministerio de Salud de Colombia ${ }^{7}$, según el cual, el $43.7 \%$ de las mujeres de 35 o más años, dicen saber hacerse el auto examen de mama y el $39.8 \%$ se lo hacen alguna vez. De las que afirman saber hacerse el auto examen, el 58.2\% responde que se lo hace varias veces al año, el $28.3 \%$ una vez al mes, el 13.5\% una vez al año y el $9.7 \%$ nunca se lo hace.

De otro lado, la mamografía es un examen poco frecuente entre las mujeres de 50 o más años, en las que apenas alcanza una prevalencia del 10\%. En esta población durante el último año, el $38.9 \%$ se lo realizó y el $53.0 \%$ se lo practica con una periodicidad de 1 a 5 años. El 72\% de las mamografías se realizaron en mujeres motivadas por la presencia de quistes o porque pensaron que con ello prevenían el cáncer; en las mayores de 50 años la mamografía fue inducida por el médico ${ }^{8}$. Incluso, algunos estudios muestran poca sensibilización de los profesionales de la salud hacia la detección precoz del cáncer de mama, a pesar de que esta estrategia continua siendo clave para controlar la enfermedad ${ }^{9}$. Merece la pena resaltar que la realización de la mamografía en Colombia está sujeta a políticas Nacionales y según la Resolución Número 00412 del 2000 sólo es obligatoria a partir de los 50 años $^{10}$.

Por otra parte, el acceso a los servicios hospitalarios en Colombia no es equitativo ${ }^{11}$, específicamente entre los afiliados al régimen contributivo y el subsidiado; para el caso de la mamografía, mientras que el $48 \%$ de los pacientes públicos se la practican, entre los privados es de $87 \%^{12}$ y peor aún, este examen no está incluido en el POS del régimen subsidiado (Plan Obligatorio de Salud Subsidiado).

El aseguramiento en salud no significa acceso real o utilización efectiva de los servicios, existe una dispendiosa tramitología para acceder a ellos, por

6. Crane, R. «Cáncer de mama.» En: S. E. Otto (Ed). Enfermería oncológica. Barcelona: Harcourt. (2003).

7. MINISTERIO DE SALUD DE COLOMBIA. Op. cit. p. 31

8. bid. p. 12.

9. Arango Ruiz, A. L., Botero Jiménez, B. H., Ossa Salazar, L. M. «Algunos cambios en la calidad de vida manifestados por varias pacientes con cáncer de mama usuarias de la EPS Seguro Social, Medellín 1998.» [Investigación de especialización] Medellín: Pontificia Universidad Javeriana, Universidad de Antioquia, facultad de Enfermería; 1998. p. 28.

10. MINISTERIO DE SALUD DE COLOMBIA. «Normas técnicas para la detección temprana del cáncer de seno. Resolución Número 00412 DE 2000.: (2000)

11. Mejía, A., Sánchez, A. \& TAmayo, J. «Equidad en el acceso a servicios de salud en Antioquia, Colombia.» Rev. Salud Pública, 9:1 (2007), p 26-38.

12. bid. p. 27. 
lo que las mujeres deciden no volver ${ }^{13},{ }^{14}$. Un estudio realizado por el Departamento Nacional de Planeación afirma que las mayores inequidades se presentan con exámenes como la mamografía ${ }^{15}$ por lo que las prácticas de autocuidado como se sugieren con este examen se hacen más complejas de lo que nuestro estudio permite explorar.

\section{Representaciones sociales}

Las representaciones sociales de una enfermedad son determinantes de los comportamientos culturales con respecto a la misma, en ellas confluyen conocimientos científicos y populares, valores y otros elementos culturales como las creencias que determinan la reacción de la sociedad y la particular de cada individuo frente a una enfermedad ${ }^{16}$. Las creencias sobre el cáncer son variadas; en algunos casos es asociado con muerte, dolor y fetide $z^{17}$.

El concepto de auto cuidado en este estudio se basa en teóricos. Para Collière, cuidar es «....un acto de vida que permite que la vida continúe y se desarrolle y, de ese modo, luchar contra la muerte; también,... es mantener la vida asegurando la satisfacción de un conjunto de necesidades indispensables para la vida» ${ }^{18}$. Para Orem, el auto cuidado es la contribución de la persona a su propia existencia, es una actividad aprendida y orientada hacia un objetivo para regular los factores que afectan su propio desarrollo y funcionamiento en beneficio de su vida, salud o bienestar ${ }^{19}$. Según Illich, los seres humanos necesitan autonomía en el auto cuidado y derecho a definir la salud e imponer límites ${ }^{20}$. Un $75 \%$ de las actividades de salud son de auto cuidado; las personas son responsables de su salud, al escoger estilos de vida, conocimientos y creencias, sin dejar de reconocer que la salud y la enfermedad son fenómenos

13. Wiesner C., Vejarano M., Caicedo J., Tovar S., Cendales R. «Cervical cytology in Soacha, Colombia: social representation, barriers and motivation.» Revista. Salud Pública, 8 (2006), pp. 185-196.

14. ECHEVERRI E. «La salud en Colombia: abriendo el siglo... y la brecha de las inequidades.» Revista. Gerencia y Políticas de Salud [online], 3 (2002), pp. 76-94.

15. Flórez C, y ACOSta O. «Acerca de su estudio Avances y desafíos de la equidad en el sistema de salud colombiano.» Fundación Corona, Instituto Nacional de Planeación, Universidad de los Andes, Universidad del Rosario. 2007.

16. Jodelet, D, y Guerrero, A Develando la Cultura. $1^{\text {a }}$ ed. México: Universidad Nacional Autónoma de México. (2000).

17. ARANGO, A., y otros. Op. cit. p 28.

18. Colliere MF. Promover la vida. Madrid, MacGraw-Hill, 1993, p. 5.

19. Marriner T, \& Alligood R. «Orem: Teoría del deficit de autocuidado.» En: Modelos y teorías en enfermería. Madrid, Hancourt, 1999. p. 177.

20. Illich I. Nemesis médica, New York, Pantheon Books, 1976, p. 265.

Feminismo/s 18, diciembre 2011, pp. 249-270 
complejos que incluyen factores no controlables, como la herencia y las condiciones situacionales y ambientales ${ }^{21}$.

En este marco, el grupo de investigación Salud de las Mujeres de la Facultad de Enfermería de la Universidad de Antioquia, se propuso emprender un estudio cualitativo sobre representaciones sociales de las mamas y del cáncer de mama que permitiera responder a la pregunta: ¿Cuáles son las representaciones sociales de las mujeres adultas sobre las mamas y su influencia en las prácticas de auto cuidado para la prevención del cáncer de mama? Para desarrollar esta pregunta nos proponemos los siguientes objetivos:

1. Comprender las representaciones sociales que las mujeres tienen sobre sus mamas y el cáncer de mama.

2. Reflexionar acerca de la influencia de estas representaciones en el auto cuidado de las mujeres y la detección temprana del cáncer.

\section{Metodología}

Este estudio se realizó en el departamento de Antioquia- Colombia, durante 12 meses entre 2006-2007, con una perspectiva metodológica cualitativa, con 19 mujeres mayores de 18 años, sin diagnóstico de cáncer de mama, con capacidad para hablar y que, voluntariamente estuvieran dispuestas a compartir sus pensamientos. A las participantes se les garantizó el manejo ético de la información.

Se utilizó la técnica de máxima variación, la cual sugiere incluir mujeres con la mayor diversidad cultural, étnica, social y económica a fin de responder a la heterogeneidad de la población ${ }^{22}$. Para determinar el número de participantes se utilizó el muestreo teórico ${ }^{23}$ es decir, partiendo de los conceptos identificados como relevantes para la comprensión del fenómeno ${ }^{24}$.

La técnica de recogida de información fue la entrevista semiestructurada ${ }^{25}$ la cual supone encuentros cara a cara entre las participantes y las investigadoras y para el análisis se siguieron los lineamientos de la teoría fundada ${ }^{26}$.

21. STEIGER, N. \& LIPSON, J. Self care-nursing. theory \& practice. Maryland: Prentice Hall Publishing. 1985.

22. Patton, M. Qualitative Research \& Evaluation Methods. Thousand Oks, Sage Publications. (2001)

23. Strauss, A., \& Corbin, J. Bases de la investigación cualitativa: Técnicas y procedimientos para desarrollar la teoría fundamentada. Medellín: Universidad de Antioquia. (2002) p. 341.

24. Ibid. p. 219.

25. Ibid. p. 224.

26. Ibid. p. 80. 
La recogida de información, el análisis y la postura teórica se hicieron conjuntamente y mantuvieron una relación reciproca durante todo el proceso investigativo.

Dos entrevistas constituyeron el estudio exploratorio, en el cual se comprobó que las preguntas y el acercamiento a las mujeres eran adecuados; la entrevista fue modificada para ampliar y enfocar los objetos de estudio. El análisis se realizó con el Software Atlas-ti y su calidad está regida por los criterios de credibilidad y veracidad ${ }^{27}$. La credibilidad se logra cuando las categorías emergen de manera natural, se saturan y son validadas por los informantes del estudio ${ }^{28}$. Para aumentar la credibilidad, los datos se les presentaron a las participantes para validar los hallazgos y se discutieron con el grupo de expertas del Grupo de Investigación Salud de las Mujeres de la Universidad de Antioquia, quienes supervisaron la construcción de las categorías principales ${ }^{29}$.

\section{Resultados}

Los datos obtenidos de 19 entrevistas a mujeres entre los 18 y 69 años de edad, con diversas características étnicas, educativas, ocupacionales y socioeconómicas permitieron identificar tres categorías de análisis: representaciones del cáncer, del autocuidado y del cuerpo y la imagen corporal. Cada categoría sirvió de base para tres publicaciones.

El articulo sobre representaciones del cáncer muestra que algunas mujeres tienen representaciones fisiológicas del cáncer, otras lo hacen desde sus efectos sociales y psicológicos e identifican sus causas con problemas personales, afectivos o hábitos cotidianos como alimentos inadecuados - «una venganza del cuerpo por los abusos que se hacen sobre el». Otros asocian la palabra «cáncer de mama» a muerte inevitable, terror, sufrimiento, devastación, incurabilidad, impotencia y dolor. La persistencia de las imágenes negativas del cáncer, se asocia al «mito de los estilos de vida» ${ }^{30}$, que responsabilizan al paciente. Este lleva a un reduccionismo biológico que oculta factores ambientales, sociales y políticos. Como lo expresa Lock la sociedad está obsesionada con los peligros y su control ${ }^{31}$, y añadimos a estos mensajes, imágenes

27. TAYLOR, S. y BOGDAN, R. Introducción a los métodos cualitativos de investigación. Barcelona, Paidós Ibérica, (1992).

28. LAGOUEYTE, M. «La citología vaginal mirada desde las mujeres». Investigación y Educación en Enfermeria, 20 (2002), pp. 58-69.

29. LINCOLN, Y. \& GUBA E. Naturalistic Inquiry. Sage Publication, (1985).

30. Medina, A. M. «Representaciones del cáncer en estudiantes de medicina: implicaciones para la práctica y la enseñanza». Revista de Salud Pública, 9(2007), pp. 369-378.

31. LoCK, M. «Breast cancer: reading the omens.» Anthropology Today, 14(1998), pp. 7-16.

Feminismo/s 18, diciembre 2011, pp. 249-270 
poderosas como aquella que «una en nueve mujeres desarrollarán el cáncer de mama» para fomentar la propia responsabilidad ${ }^{32}$, de esa forma, se magnifican los fantasmas del cáncer que nuestras sociedades en desarrollo están tratando de superar.

El artículo de representaciones sociales y prácticas de auto cuidado para la prevención del cáncer de mama, muestra que el auto examen, mamografía y examen clínico, no coinciden con los parámetros de tamizaje para la detección precoz. Por el contrario, hacen énfasis en la dimensión del embellecimiento de las mamas. Este hallazgo tan contundente y perturbador permite entender que el cáncer de mama tiene fuertes representaciones sociales debido a las severas implicaciones en el aspecto atractivo y autoimagen de la mujer; por esto, desarrollamos este articulo donde mostramos cómo el cuerpo y la imagen corporal median las representaciones del cáncer da mama.

Para las mujeres del estudio, las mamas constituyen un objeto de atracción para el sexo opuesto y en ese sentido le dan un alto valor estético y erótico. Se representan como un objeto perfectible sobre el cual deben ejercer cuidados para mantenerse bellas dentro de los estándares de la moda, en lo que invierten gran cantidad de atención, dinero y tiempo. Para otras, las mamas representan además, la tradicional función materna de afecto y alimentación.

Para las participantes las mamas son muy importantes, no se conciben sin ellas, no importa que el tamaño de las mamas sea grande o pequeño, pero nunca sin ellas. Las mamas marcan la diferencia de sexo, son elemento fundamental de la identidad femenina. Las reconocen con una doble función, como vínculo con los hijos y como parte de la sexualidad; como expresa Laura ( $\mathrm{R}$ 159): «Importante en el lado de los hijos, me parece que es un vínculo que se debe tener con los hijos; para la parte sexual es importante». A estas mujeres les preocupa el impacto negativo que el cáncer de mama pueda tener sobre la estética corporal, lo que sería «doloroso y difícil de aceptar», de ahí que consideren las pérdidas corporales, ocasionadas por la enfermedad, como difíciles de llevar, ya que afectan la autoestima y comprometen la autoimagen y la confianza para cautivar al sexo opuesto. Según estas mujeres, en el contexto en que vivimos, el énfasis de las mamas se centra en su valor sexual y estético, se las considera "puro adorno» $\mathrm{y}$ «decoración... nos hace ver más hermosas» (Morelia R 22).

Por el contrario, para las indígenas Emberá, las mamas cobran importancia a partir de la vivencia de la maternidad, al dar la posibilidad de amamantar

32. LoCK, M. Op cit, pp. 7-16. 
a los hijos, privilegio sólo de las hembras humanas; les parece algo «mágico»y les produce mucha gratificación su capacidad fisiológica para aportar al crecimiento de otro ser humano, como lo expresa Julieta (R12), mujer del Resguardo Indígena de Cristianía (Jardín Antioquia): «es muy bonito dar algo del cuerpo que es la leche, y sólo la da la hembra, yo sé que no todas miran así, ni ven esa magia, que bonito que yo estoy dando algo de mí, estoy haciendo crecer a otro ser con mis senos, de ahí está saliendo la leche».

\section{Discusión}

\subsection{Asumiendo o no los estereotipos}

Encontramos que las mujeres construyen su imagen corporal de diversas formas. Por un lado, aquellas que autoaplican estereotipos construidos por agentes externos y por otro, las que se apartan de tales estereotipos y asumen su propia figura corporal.

Al realizar el estudio sobre la auto aplicación de estereotipos, se encontró que la satisfacción con la imagen corporal depende de las pautas estéticas preestablecidas. Tener un cuerpo agradable es una exigencia social impuesta por los medios masivos de comunicación, las mamas, según esto, se constituyen en un objeto estético y erótico. Para muchas, los medios son «los culpables» (Laura R 504), pues tienen el poder para interferir la confianza de las mujeres respecto a su imagen corporal. El interés general de los medios no es mejorar la situación de las mujeres, por el contrario, son ellas con su cuerpo quienes le prestan un servicio bastante lucrativo a los medios y al mercadeo; así lo expresa Laura ( $\mathrm{R} 476$ ): «No hay casi ningún producto que no tenga a una mujer involucrada [en la publicidad], sea un tarro de pintura, siempre muestran a una mujer en bikini mostrando los senos» El objetivo de los medios es promocionar el consumo a través de la figura femenina, situación que impacta negativamente la identidad de las mujeres en tanto se alejan de ser sí mismas. La importancia del cuerpo está en las mamas grandes, pues así son más visibilizadas y «atraen al sexo opuesto» (Laura R 34).

Para el mundo publicitario el cuerpo de las mujeres es el botín de la publicidad, ésta ha construido las pautas estéticas que permiten mediatizar positivamente la atracción de consumidores, capturando la atención sobre «miles de productos» en el mercado, lo que requiere de mujeres con las medidas perfectas para tal fin. El estereotipo mencionado hace parte del referente social que tienen mujeres y hombres durante los procesos de individuación-socialización para constituir la identidad y la autoimagen a través de la imitación. La influencia de tales modelos induce a las mujeres a desear para sí, mamas 
grandes para sentirse «orgullosas» y «exhibirlas». Por el contrario, quienes tienen mamas pequeñas, se sienten «incómodas» y evitan la exposición del cuerpo; si tuvieran recursos se harían cirugía; Hellen expresa (R 14): «Yo tengo varias amigas que..., se han puesto siliconas y... se sienten muy orgullosas; se ponen escotes y todo eso, pero cuando son así pequeñitas y todo se sienten como incómodas no les gusta exhibirlas»

Mantenerse dentro de los estereotipos aceptados de belleza, puede implicarle a las mujeres el riesgo de explotación del mercado en búsqueda del tamaño perfecto: «Todas se quieren hacer un implante de siliconas jah!... existe una crema, incluso, nos dejamos engañar por lo que dicen los medios, que tal producto sirve para eso» (Laura R 550).

El valor que las mujeres les dan a las mamas está sujeto a la concepción del cuerpo a partir de la autoaplicación de estereotipos construidos por otros. En el auto concepto incorporan el modelo, pero, cuando el cuerpo físicamente no logra el esquema ideal, se torna en un problema, "iAh no, es que yo no me sentía bien!» No... ¡Muy triste! Si me voy a poner un vestido de baño, no es lo mismo» (Laura, R 575). Lo que precisa de las mujeres un trabajo sobre su figura.

Para estas mujeres, el problema está en que los patrones de belleza los determinan agentes externos a ellas mismas, quedando sujetas a ciertos estereotipos que constituyen la «carta de presentación» (Laura R 534): ser joven, armoniosa, voluptuosa, mamas bien firmes, desarrolladas y grandes. Laura ( $\mathrm{R}$ 590) dice: «la imagen la han vendido los medios, la necesidad parte es de formas estéticas que reproducen los medios de quien, en realidad, sale la necesidad de mostrar unos cuerpos voluptuosos». La moda arrastra a muchas mujeres sin importar lo que proponga, induciendo a la cirugía cosmética de las mamas, ya sea para aumentar o disminuir, y así acercarse al modelo ideal.

Las mujeres, socializadas como seres no para sí, sino para el otro, tienen incorporada la necesidad de ser conquistadas, entonces, para tener un lugar en el mundo del otro requieren de unas condiciones estéticas que atrapen las miradas, así logran existir y ser alguien. Lo que explica que en algunas ocasiones, se da prioridad al cuidado estético de las mamas por encima de la salud. Este comportamiento es potenciado por la educación impartida a las mujeres en la que se insiste en la idea de que son las mujeres quienes deben mantener la relación de pareja y para ello es necesario estar siempre atractiva dentro del paradigma ideal, en el cual, el tamaño de las mamas tiene un valor erótico para los hombres. En este contexto, una de las alternativas para complacer al compañero sexual es hacerse mamoplastia de aumento. 
Según las entrevistadas, la inconformidad con las mamas y el deseo de operarse también se trasmite entre pares y de generación en generación como lo expresa Liliana (R22): «De pronto, igual, yo creo también que el inconformismo empezó porque yo vi que mi mamá se operó, entonces yo, ya, ¿por qué ella y por qué yo no?». La autoaplicación de estereotipos implica asumir que las mamas grandes son la carta de presentación ante los demás; quienes las tienen pequeñas sufren de baja autoestima, Morelia ( $\mathrm{R} 22$ ) dice: «Las mujeres sienten que sus senos son un tipo de presentación, o sea, actualmente a mí me parece que sea así, porque una mujer... que tiene los senos pequeños se siente acomplejada».

En este sentido, las mujeres ven la necesidad de que se de educación en salud desde la infancia y la adolescencia; según ellas, se carece de acompañamiento para estimular el reconocimiento corporal durante este periodo, lo que es importante para sentirse bien con el tamaño de sus mamas; pues hay niñas que "se preocupan porque casi no les han crecido. Es una tablita por completo» (Doris R 4).

Sin embargo, los patrones estéticos imperantes no son aceptados por todas las mujeres; algunas se apartan de estereotipos, su referente es el buen funcionamiento del cuerpo, les basta con reconocer que funciona normalmente y no tiene deformidades, «uno debe estar agradecido con dios con lo que me dio, que me lo dio normal, que no tiene deformidades, nada raro» (Beatriz R16). Para ellas la auto aceptación es un indicador de auto estima, aunque sus formas corporales no se acerquen a las pautas de belleza expuestas por los medios, reconocen su cuerpo, lo aprecian y lo asumen al margen de influencias externas; no conciben ser admiradas por algo que no es propio, así lo refiere Bibiana (R 48): «Yo así como estoy me siento bien..., yo no quiero que me estén... catalogando por algo que no es mío... no tengo por qué estar aparentando... Lo que tengo, lo tengo, si les gusta bien y si no también». Rechazan que las mujeres aumenten sus mamas por complacer a los hombres, sostienen que «si me quiere, me acepta como soy» (Morelia R 74).

La auto aceptación es un proceso que involucra la superación de los estereotipos, aunque alguna mujeres pasan por altibajos, «al principio, cuando empecé en el modelaje, sí quería como operarme el busto porque yo veía que todo el mundo, pues que los contratos surgían más del busto, pero después se me fue pasando como la bobada. Ya no me importó» (Bibiana R 10). Es un intento por mantener la propia valía, desechando modelos, para llegar, finalmente, a ser sí misma. «no soy como una modelo, pero sí, me siento bien con la imagen corporal (Flor R 10). 
Los hallazgos frente a las representaciones del cáncer, el auto cuidado y la imagen corporal, que son las categorías mayores en este estudio, se validaron con las participantes. A la primera participante le preocuparon dos asuntos, la insensibilidad frente esta problemática y el cómo acceder a los pueblos indígenas con un lenguaje comprensible frente a este tema; reconocía que las mamas nos representan, como son utilizadas y seguirán siendo utilizadas. A la participante dos le preocupaba que se estigmatizara a alguien por no cuidarse y que la identidad de la mujer se afecte con todo tipo de estereotipos, afirma además, que una mujer no deja de ser mujer por seguir estos estereotipos, sin embargo sabia que seguirlos significa hacerse daño a si misma. A la participante tres le preocupa cómo mejorar la salud de las mujeres. La cuatro quisiera que los hombres cercanos a las mujeres entendieran más sobre este tema y que el miedo al cáncer no radique en el hecho de que les van a quitar un seno. La participante cinco señalaba que las mujeres deben hacerse conscientes de que un implante mamario no garantiza un hombre a su lado; además que la gente debe conocer los efectos que tiene esta cirugía a largo plazo y que se necesitan más campañas sobre el tema. La seis dijo que la responsabilidad de género está en que no solo velemos por la salud de los demás, sino en exigirle al estado las condiciones de vida digna y agradeció a las investigadoras por presentarle los resultados.

\subsection{Belleza como modelo del ser femenino}

No es gratuito que dos novelas de la mayor importancia comercial y social en Colombia tengan como asunto central la belleza. Estas novelas, aunque trivializadas por la academia, son un buen pulso de lo que es preeminente a una cultura y la expresión de representaciones sociales colectivas. Ivan Ulchur ${ }^{33}$, hace la semblanza de una Betty en Betty la Fea, la mujer que encarna un antimodelo de belleza por su apariencia física y sus pobres habilidades sociales y femeninas para la conquista, pero es brillante y bien educada, habilidades que son constantemente olvidadas en un mundo de hombres y mujeres atractivos, exitosos y deseables que la miran con desprecio. Lamentablemente en la novela, Betty debe despojarse de su fealdad para poder ser aceptada en una relación romántica ${ }^{34}$ con lo que la novela perpetúa el estereotipo de que la belleza sigue siendo, a fin de cuentas, la puerta de entrada en nuestra sociedad ${ }^{35}$.

33. Ulchur, I. «Betty la fea: la suerte de la inteligencia». Chasqui, 71 (2000) http://chasqui. comunica.org/ulchur71.htm Consultado el 23-04-2008.

34. Ulchur, I. Op. Cit. 1.

35. Ibíd. p. 1. 
Por su parte, Sin Tetas No Hay Paraíso es una historia de la vida real, basada en el libro de Gustavo Bolívar, donde las protagonistas son dos mujeres jóvenes que sueñan con ascender en la escala social y utilizan sus cuerpos y la belleza artificial para hacerlo. Igualmente, con esta serie se trató de llamar la atención de la sociedad sobre la proliferación de implantes mamarios sin las observaciones médicas requeridas y sobre las llamadas «chicas prepago», donde la falta de educación, la ignorancia y la necesidad de conseguir dinero hacen que las jóvenes caigan en un medio donde su cuerpo es la única opción para alcanzar sus metas ${ }^{36}$.

La preocupación de las mujeres por su apariencia física ha sido extensamente revisada en los países desarrollados, particularmente en los Estados Unidos, país en el que nosotros, los colombianos, nos miramos. En nuestro medio los estándares de belleza se han homogenizado tratando de seguir los norteamericanos y Europeos.

El cuerpo es crucial en la concepción actual de género, las mujeres contemporáneas afrontan intensas presiones para cumplir con ciertos ideales de belleza, y hacen una gran inversión emocional, física y financiera en su cuerpo. Para esta autora, las mujeres tienen relaciones problemáticas con su cuerpo con mayor frecuencia que los hombres ${ }^{37}$. Con el objeto de ser más atractivas, muchas mujeres a través de la historia, han efectuado rutinas, rituales y alteraciones corporales, han vendado sus pies, usado corsés, fajas, tatuajes, pircings, implantado gemas en sus cuerpos y una larga lista da acciones encaminadas a mejorar la apariencia de su cuerpo. Estos rituales han transcendido las clases, las razas y las regiones ${ }^{38}$. Las mujeres del estudio están sujetas a estas presiones, excepto las indígenas, cuyo modelo de belleza no corresponde al modelo hegemónico occidental.

\subsection{Imagen Corporal y Cuidados del Cuerpo}

En los países Occidentales, autores de las ciencias sociales han investigado y teorizado sobre los efectos en la salud de la tendencia esteticista del cuidado, dos asuntos centrales en la discusión de nuestros hallazgos. Algunos de ellos afirman que las personas desarrollan estilos de vida saludables para preservar

36. Wikipedia. Sin tetas no hay paraíso < http://es.wikipedia.org/wiki/Sin_tetas_no_hay_ para\%C3\%ADso_(Colombia) > Consultado el 16-03-2011.

37. Gimlin, D. Body work: Beauty and self-image in American culture. Berkeley University of California Press. (2001).

38. Banner, L. American Beauty. Los Angeles: Figueroa Press 2 (2005). p. 548.

Feminismo/s 18, diciembre 2011, pp. 249-270 
la belleza y la apariencia joven, en lugar de pensar en su salud ${ }^{39}$. Las personas muchas veces se envuelven en prácticas que no son saludables para ser físicamente más aceptables, por lo que ha aumentado el número de cirugías cosméticas, blanqueamientos del la piel o ingestión de cantidad de hormonas, vitaminas, suplementos y esteroides ${ }^{40}$. La percepción del cuerpo propio influye en el consumo de servicios y productos para mantener la imagen como hemos encontrado en nuestros resultados ${ }^{41}$; no obstante, Black, cree que la salud vista desde una perspectiva más holística permite entender cómo las terapias de embellecimiento pueden mejorar la salud física y emocional de la persona $^{42}$.

La preocupación y el deseo por cambiar la apariencia de las mamas en nuestras entrevistadas puede ser teorizada desde las reflexiones de Schouten, quien desde la perspectiva antropológica del comportamiento encontró que las cirugía plástica en hombres y mujeres son ritos personales de paso y de reconstrucción de la identidad ${ }^{43}$. Prácticas como estas, explotan los estándares no reales de belleza donde se idealiza una versión particular de mujer. El problema más grave, es que las personas no cuestionan seriamente las construcciones de belleza con su carácter opresivo ${ }^{44}$, como sucedió con nuestras entrevistadas.

\subsection{Discriminación Basada en la Apariencia}

Denunciamos la discriminación basada en la apariencia, de la cual la mayoría de las mujeres son poco conscientes. Con respeto a esto Berry afirma que es el último bastión de los prejuicios socialmente aceptados, es decir, que para la mayoría de las personas no causa rechazo ${ }^{45}$. Para esta autora, la inequidad social está relacionada con los prejuicios y la discriminación basada en la

39. UCOK, O. «The fashioned survivor institutionalized representations of women with breast cancer» Communication and Medicine, 4 (2007), pp. 67-78.

40. BerRY, B. Beauty bias: Discrimination and social power Estates Unites. Praeger.1 (2007) p 163.

41. ThOMPSON, C. \& Hirchman, E. «Understanding the socialized body: A poststructuralist analysis of consumers' self-concepts, body images and self care practices». The journal of consumer research, 22 (1995), pp. 139-153.

42. BLACK, P. Beauty industry: gender culture and pleasure London, New York, Taylor and Francis. (2004) p. 214.

43. SCHOUTEN, JW. «Selves in transition: Symbolic consumption in personal rites of passages and identity reconstruction». The journal of consumer research, 17(1991), pp. 412-425.

44. HEVORY, T. Body/politics in reproduction and production and reconstruction. Westport, CT Praeger. (2000).

45. BERRY, B. Op.Cit p. ix. 
apariencia física; y considera que rasgos como la raza, el género, la edad y los recursos económicos, formas de discriminación mas estudiados, están sobrepuestos con todas estas categorías como el peso, la estatura, la forma de los ojos, la incapacidad, la deformidad, carencia de dientes y la vejez, que son importantes marcadores del trato discriminatorio laboral, afectivo, amistoso entre otros ${ }^{46}$.

En el mundo del trabajo el aspecto físico o el atractivo favorecen a la persona, mientras que los que son poco atractivos pierden oportunidades y las ventajas que generosamente son otorgadas a los primeros. Afirman también que muchos empleadores se preocupan por el aspecto físico de sus empleados y hasta toman decisiones de empleo, basadas en el aspecto físico de los candidatos ${ }^{47}$.

Hamermesh y Biddle ${ }^{48}$ fueron un paso mas allá de Corbett $^{49}$, ellos diseñaron una serie de experimentos para medir en forma objetiva la discriminación basada en la apariencia y examinaron el impacto en los ingresos, encontrando que la gente común gana menos que el promedio de las personas bien parecidas, la deducción es ligeramente mayor del 5 a $10 \%{ }^{50}$.

Las personas atractivas tienen como capital su apariencia, y tienen mejores precios en el mercado del noviazgo y el matrimonio, al punto que las mujeres bellas se casan con hombres más prestigiosos y con mayores ingresos que ellas. Las mujeres y los hombres atractivos son más populares y tienen más $\operatorname{citas}^{51}$. Situación que podría explicar el temor de nuestras entrevistadas a una mastectomía ya que pudiera llevarlas a la imposibilidad de conseguir compañero sexual o esposo, o a un abandono por parte de ellos.

Las mujeres tienen que someterse a un doble estándar en la apariencia que no se impone con tanto rigor sobre los hombres ${ }^{52}$ y deben realizar gran cantidad de trabajo corporal para conseguir un ideal de belleza inalcanzable ${ }^{53}$,

46. Ibíd. p. ix.

47. CORBETt, W. «The ugly truth about appearance discrimination and the beauty of our employment discrimination law». Duke Journal of Gender Law \& Policy (2007) http:// goliath.ecnext.com/coms2/gi_0199-6416718/The-ugly-truth-about-appearance.html Consultado el 5-05-2008.

48. Hamermesh, D., \& Biddle, J. «Beauty and the Labor Market». The American Economic Review. 84 (1994), pp. 1174-1194.

49. Corbett, W. Op. Cit.

50. Hamermesh, D., \& Biddle, J. Op. Cit., pp. 1174-1194.

51. BERRY, B.. Beauty bias: Discrimination and social power. Estates Unites. Praeger.1 (2007) p. 29.

52. GIMLIN, D. Body work: Beauty and self-image in American culture. Berkeley University of California Press. (2001). P. 4.

53. Ibid. p. 4.

Feminismo/s 18, diciembre 2011, pp. 249-270 
trabajo que parece reemplazar las prácticas de auto cuidado frente a la prevención del cáncer de mama.

Las entrevistadas hablan de un interés por la mamoplastia, igual a lo ocurrido en los Estados Unidos hace algunas décadas que obligó a algunas investigadoras realizar estudios prospectivos y retrospectivos sobre este problema. Otros trabajos muestran que para las mujeres, las cirugías de mama obedecen a condiciones específicas de presión en términos de que valdrían más, serian más amadas y atendidas si se adherían a las normas culturales de femini$\operatorname{dad}^{54,55,56}$.

Saffon investigadora del Centro de Estudios de Derecho, Justicia y Sociedad, ha escrito sobre la discriminación de los feos en Colombia, ella considera que es un fenómeno global; y al igual que Berry considera que es un tipo de discriminación más sutil y más difícil de identificar, denunciar y evitar. Para ella "estética funciona como un poderoso mecanismo de diferenciación social ${ }^{57}$.

Saffon $^{58}$ argumenta que nuestra sociedad estratificada hace que aquellos que pertenecen a clases de mayor élite, prestigio y poder y que Mills llamó la elite del poder ${ }^{59}$ son quienes usualmente fijan los estándares de apariencia física y es a partir de estos estándares, como se discrimina a un miembro de una clase social menos aventajada, o que no tiene la apariencia que satisface a esa clase dominante, adicionalmente considera que no solo la apariencia se usa como forma de discriminación sino también los gustos y modales

\subsection{Cáncer de mama e Imagen Corporal}

Nuestra discusión ha retomado extensamente los hallazgos en relación con los imaginarios, prácticas y percepciones de las mujeres sobre las conductas de autocuidado. Al hablar de cuidado ellas también hacen elaboraciones acerca de los cuidados que requieren una vez comienzan a padecer de cáncer. Quizás los fantasmas que más preocupan a la mujer, son la mastectomia, la alteración de su imagen corporal y los efectos sobre la vida sexual; para casi todas era una preocupación común.

54. ZimmermanN, S. Silicone survivors: Women's experiences with breast implants Temple University Press. (1998).

55. DAVIS, K. Reshaping the Female Body: The Dilemma of Cosmetic Surgery. New York, Routledge. (1995)

56. BORDO, S. Unbearable Weight: Feminism, Western Culture, and the Body. Berkeley University of California Press. (1993).

57. SAFFon, M. «La discriminación de los feos». Semana.Com. (2006). <http://www.semana.com/wf_InfoArticulo.aspx?idArt=93778> Consultado el 16-03-2011.

58. Ibíd.

59. Milss W. The Power Elite, Oxford Press, (1956). 
Como ninguna de nuestras entrevistadas tenía cáncer de mama, privilegiamos el testimonio de Raven Light quien escribió su experiencia personal. Ella era una modelo de 31 años que en la primavera del 91, en un lapso de tres días, se le diagnosticó cáncer de mama cuyo tratamiento consistió en la amputación de la mama derecha, dejándole una cicatriz de 33 puntos. Tres semanas después de la amputación se enfrentó a la quimioterapia que le hizo perder su hermoso pelo rojo, veinte libras de un cuerpo que ya era delgado, sus cejas, sus pestañas, el vello púbico y encima de todo esto la libido ${ }^{60}$.

Ella se preguntaba cómo afrontarlo, ya que el mirarse al espejo se convertía en un proceso de tortura diario; los tres años siguientes fueron una lucha constante para reconstruir una relación positiva con su cuerpo y reintegrarla en concepto de quién es ella - que no es sólo su cuerpo, pero su cuerpo está dentro de ella. Aunque ha reanudado el modelaje y ha permitido a miles de personas ver su belleza, incluyendo sus cicatrices en la intimidad de su cuarto, su cuerpo la tortura. Ella se cuestiona si otras mujeres podrían tener sentimientos similares sobre sus cuerpos alterados ${ }^{61}$.

Un estudio que utilizó la hermenéutica cualitativa Heideggerian realizado por Boehmke y Dickerson ${ }^{62}$ llamado El Diagnóstico de Cáncer de Mama, la Transición de Salud a Enfermedad es vital para el entendimiento de lo que representa sufrir el cáncer, y aunque en ese caso las participantes eran mujeres con cáncer, su punto de vista fue reproducido por nuestras entrevistadas, quienes fueron bastante intuitivas en sus representaciones de lo que es padecer un cáncer a pesar de que ninguna de ellas lo había afrontado ${ }^{63}$.

Las mujeres con cáncer de mama deben asimilar en sus vidas cicatrices físicas, efectos tóxicos de la terapia medicamentosa, angustia emocional e interrupción en la realización de la vida familiar, trabajo y roles sociales, sus reacciones incluyen sentimientos de decadencia en el funcionamiento sexual y matrimonial ${ }^{64}$; sentimientos y percepciones que fueron descritas por las entrevistadas, aunque no sufrieran de la enfermedad, las mujeres describieron el diagnóstico y la experiencia del tratamiento como «una borradura de un antiguo yo» debido a las modificaciones físicas y emocionales que provoca, pues la cirugía y la terapia cambian la visión femenina no sólo de sus cuerpos,

60. Brittingham, D. \& Light, R. «Breast Cancer and Body Image», Off Our Backs xxiv, 10, 11 (November 1994). 1

61. Ibid. P. 1.

62. Boenmke, M., \& Dickerson, S., «The Diagnosis of Breast Cancer: Transition From Health to Illness» Oncology Nursing Forum. 33 (2006). pp. 11-21.

63. BOeHMke, M., \& DiCKerson, S. Op Cit. pp. 11-21.

64. Ibíd. Pp. 11-21. 
sino de su misma identidad, al punto de considerar que después de la cirugía nunca serían la misma persona de antes ${ }^{65}$.

Según la perspectiva actual, la imagen corporal es una construcción que se refiere a lo que uno piensa, siente, percibe y hace en relación con su propio cuerpo, como una parte del auto concepto ${ }^{66}$. Aunque la definición del término auto concepto varía, es entendido como la sumatoria de las percepciones o referencias que las personas tienen de ellas mismas y que incluyen juicios en el comportamiento, habilidades o apariencia física ${ }^{67}$.

En ese sentido, algunos autores estudiaron la imagen corporal y su relación con la auto estima en una muestra de mujeres españolas con cáncer de mama en etapa temprana, y encontraron que la mayoría sufre repercusiones físicas significativas, que influyen en forma negativa en la imagen que la paciente tiene de su cuerpo ${ }^{68}$ además de los desórdenes afectivos y cambios en sus sexualidad y autoestima ${ }^{69}$. Dentro de los diferentes tipos de cáncer, el de mama sobresale por su mayor efecto en la imagen corporal ${ }^{70}$.

La autoestima es otro término relacionado con la imagen corporal, la cual es considerada una actitud positiva o negativa hacia el yo, basada en la evaluación de sus características e incluye sentimientos de satisfacción consigo mismo ${ }^{71}$. Para muchas mujeres, la autoestima está basada exclusivamente en su imagen corporal y como consecuencia de esto su desempeño social y sus relaciones interpersonales se ven afectados ${ }^{72}$, lo que es más complicado si las mujeres sufren cambios o desfiguraciones debido a enfermedades como el cáncer. La percepción de su apariencia física, comparada con el ideal social, puede resultar en un impacto negativo para sus actitudes emocionales y su calidad de vida ${ }^{73}$.

\section{Conclusiones}

Para las mujeres del presente estudio las mamas tienen un significado fundamentalmente estético y centrado en la feminidad; las mamas son básicamente

65. Ibíd. Pp. 11-21.

66. Manos, D., Sebastián, J., Bueno, M., Mateos, N., De la Torre, A. «Body image in relation to self esteem in a sample of Spanish women with early stage breast cancer». Psicooncologia, 2(2005), pp. 103-116.

67. Manos, D., Sebastián, J., Bueno, M., Mateos, N., De la Torre. Op Cit. pp. 103-116.

68. Ibíd. pp. 103-116.

69. Ibíd. pp. 103-116.

70. Ibíd. pp. 103-11.

71. Ibíd. pp. 103-116.

72. Ibíd. pp. 103-116.

73. Ibíd. pp. 103-116. 
para la identidad sexual femenina y por lo tanto la pérdida total o parcial puede alterar la auto-imagen y la vida sexual; por estas razones el cuidado de las mismas es concebido desde lo estético y se reduce a mantenerlas bellas dentro de los estándares de la moda. En este sentido, prácticas como el auto examen de mama y la mamografía no existen en el imaginario de estas mujeres.

La discriminación basada en la apariencia, con efectos negativos sobre su economía, su salud y su desarrollo como ser humano integral, está presente en el imaginario de estas mujeres, lo que puede afectar el tratamiento en el caso de sufrir de cáncer mamario. Por lo tanto, quienes legislan en salud debe entender que existe una construcción problemática del cuerpo femenino que merece ser denunciada e investigada como un asunto político esencial en la construcción de la subjetividad femenina.

Como investigadoras sociales nos intriga el hecho de que las mujeres del estudio, sin haber padecido de cáncer, describieran con tanta vivacidad sus preocupaciones por los efectos del cáncer de mama como la autoimagen, la autoestima, la relación de pareja y la sexualidad, consideraciones que creemos, tienen que ver con la construcción problemática de la feminidad que nos ocupa y preocupa en este articulo

\section{Referencias bibliográficas}

Arango Ruiz, A. L., Botero Jiménez, B. H., Ossa Salazar, L. M. «Algunos cambios en la calidad de vida manifestados por varias pacientes con cáncer de mama usuarias de la EPS Seguro Social, Medellín 1998.» [Investigación de especialización] Medellín: Pontificia Universidad Javeriana, Universidad de Antioquia, facultad de Enfermería; 1998. p. 28

BANner, L. American Beauty. Los Angeles: Figueroa Press 2 (2005). p 548

Boenmke, M., \& Dickerson, S., «The Diagnosis of Breast Cancer: Transition From Health to Illness» Oncology Nursing Forum. 33 (2006). pp. 11-21.

BerRY, B. Beauty bias: Discrimination and social power. Estates Unites. Praeger.1 (2007) p 29

BerRY, B. Beauty bias: Discrimination and social power Estates Unites. Praeger.1 (2007) p 163.

BLACK, P. Beauty industry: gender culture and pleasure London, New York, Taylor and Francis. (2004) p 214.

BORDO, S. Unbearable Weight: Feminism, Western Culture, and the Body. Berkeley University of California Press. (1993).

Brittingham, D. \& Light, R. «Breast Cancer and Body Image», Off Our Backs xxiv, 10, 11 (November 1994). 1

COlliere, M. F. Promover la vida. Madrid, MacGraw-Hill, 1993, p. 5 
CORBETT, W. «The ugly truth about appearance discrimination and the beauty of our employment discrimination law». Duke Journal of Gender Law \& Policy (2007) http://goliath.ecnext.com/coms2/gi_0199-6416718/The-ugly-truthabout-appearance.html Consultado el 5-05-2008

Crane, R. «Cáncer de mama.» En: S. E. Otto (Ed). Enfermería oncológica. Barcelona: Harcourt. (2003).

DAVIS, K. Reshaping the Female Body: The Dilemma of Cosmetic Surgery. New York, Routledge. (195)

ECHEVERRI E. «La salud en Colombia: abriendo el siglo... y la brecha de las inequidades.» Revista. Gerencia y Políticas de Salud [online], 3 (2002), pp. 76-94.

Flórez, C. y Acosta, O. «Acerca de su estudio Avances y desafíos de la equidad en el sistema de salud colombiano.» Fundación Corona, Instituto Nacional de Planeación, Universidad de los Andes, Universidad del Rosario. 2007

GIMLin, D. Body work: Beauty and self-image in American culture. Berkeley University of California Press. (2001). P 4.

Hamermesh, D. \& Biddle, J. «Beauty and the Labor Market». The American Economic Review. 84 (1994), pp. 1174-1194

HeVOrY, T. Body/politics in reproduction and production and reconstruction. Westport, CT Praeger. (2000).

ILliCH, I. Nemesis médica, New York, Pantheon Books, 1976, p. 265.

Jodelet, D, y Guerrero, A Develando la Cultura. $1^{a}$ ed. México: Universidad Nacional Autónoma de México. (2000).

LAgoueyte, M. «La citología vaginal mirada desde las mujeres». Investigación y Educación en Enfermeria, 20 (2002), pp. 58-69.

Lincoln, Y \& GUBA, E. Naturalistic Inquiry. Sage Publication, (1985).

LoCK, M. «Breast cancer: reading the omens.» Anthropology Today, 14(1998), pp. 7-16.

Manos, D., Sebastián, J., Bueno, M., Mateos, N., De la Torre, A. «Body image in relation to self esteem in a sample of Spanish women with early stage breast cancer». Psicooncologia, 2(2005), pp. 103-116.

MARriner, T. \& Alligood, R. «Orem: Teoría del deficit de autocuidado.» En: Modelos y teorías en enfermería. Madrid, Hancourt, 1999. p. 177.

Medina, A. M. «Representaciones del cáncer en estudiantes de medicina: implicaciones para la práctica y la enseñanza». Revista de Salud Pública, 9(2007), pp. 369-378.

Mejía, A., SÁnchez, A. \& TAmayo, J. «Equidad en el acceso a servicios de salud en Antioquia, Colombia.» Rev. Salud Pública, 9:1 (2007), p 26-38.

MiLss, W. The Power Elite, Oxford Press, (1956).

MINISTERIO DE LA PROTECCIÓN SOCIAL COLOMBIA. «Política Nacional de Salud Sexual y Reproductiva.» Bogotá. El Ministerio, (2003). Documentos técnicos p 11. 
MINISTERIO DE SALUD DE COLOMBIA. «III Estudio Nacional de Salud Bucal y II Estudio Nacional de factores de riesgo de enfermedades crónicas- ENFERC II.» Colombia. El Ministerio; Serie Documentos Técnicos. (1999) p 31

MINISTERIO DE SALUD DE COLOMBIA. «Normas técnicas para la detección temprana del cáncer de seno. Resolución Número 00412 DE 2000.: (2000)

ORGANIZACIÓN MUNDIAL DE LA SALUD. «Programas Nacionales de Control del Cáncer: Políticas y Pautas para la Gestión». Organización Mundial de la Salud. <http://www.paho.org/Spanish/AD/DPC/NC/pcc-NCCP.pdf>. Consultado el 7-08-2005.

Pardo, C., Murillo, R., Piñeros, M., y Castro, M. Casos nuevos de cáncer 2003 en el Instituto Nacional de Cancerología Colombia, Revista Colombiana de Cancerología, 7 (2003). p. 5.

Patton, M. Qualitative Research \& Evaluation Methods. Thousand Oks, Sage Publications. (2001)

RAdicE, D. \& RedAelli, A. Breast cancer management: quality of life and cost considerations. Harmacoeconomics 21 (2003), pp. 383-396.

SAFFON, M. «La discriminación de los feos». Semana.Com. (2006). <http://www. semana.com/wf_InfoArticulo.aspx?idArt=93778> Consultado el 16-03-2011

Schouten, J. W. «Selves in transition: Symbolic consumption in personal rites of passages and identity reconstruction». The journal of consumer research, 17(1991), pp. 412-425.

SteIGER, N. \& LIPSON, J. Self care-nursing. theory \& practice. Maryland: Prentice Hall Publishing. 1985.

STRAUSS, A. \& CORBIN, J. Bases de la investigación cualitativa: Técnicas y procedimientos para desarrollar la teoría fundamentada. Medellín: Universidad de Antioquia. (2002) p 341

TAYLOR, S. Y BOGDAN, R. Introducción a los métodos cualitativos de investigación. Barcelona, Paidós Ibérica, (1992).

THOMPSON, C. \& HiRCHMAN, E. «Understanding the socialized body: A poststructuralist analysis of consumers' self-concepts, body images and self care practices». The journal of consumer research, 22 (1995), pp. 139-153.

UCOK, O. «The fashioned survivor institutionalized representations of women with breast cancer» Communication and Medicine, 4 (2007), pp. 67-78.

UlChur, I. «Betty la fea: la suerte de la inteligencia». Chasqui, 71 (2000) http:// chasqui.comunica.org/ulchur71.htm Consultado el 23-04-2008.

Wiesner, C., Vejarano, M., Caicedo, J., Tovar, S., Cendales, R. «Cervical cytology in Soacha, Colombia: social representation, barriers and motivation.» Revista. Salud Pública, 8 (2006), pp. 185-196.

Wikipedia. Sin tetas no hay paraíso < http://es.wikipedia.org/wiki/Sin_tetas_no_ hay_para\%C3\%ADso_(Colombia) > Consultado el 16-03-2011. 
Zimmermann, S. Silicone survivors: Women's experiences with breast implants Temple University Press. (1998).

Feminismo/s 18, diciembre 2011, pp. 249-270 